Artículo original

\title{
PROPUESTA DE FÓRMULA PARA LA DETERMINACIÓN DE LA DENSIDAD DE MUROS CONSTRUIDOS CON BLOCKER II
}

PROPOSAL FORMULA FOR THE DETERMINATION OF THE DENSITY OF WALLS BUILT WITH BLOCKER II

Dina Cotrado Flores ${ }^{1}$

Información del artículo:

Recibido: $18 / 082019$

Aceptado: 10/02/2020

${ }^{1}$ Escuela de ingeniería civil, Universidad Privada de Tacna, Tacna, Perú Correo: dcotrado@upt.edu.pe

Vol. $1, \mathrm{~N}^{\circ} 1$.

INGENIERÍA
INVESTIGA 


\section{Resumen}

Se realizó ensayos en pilas y muretes de albañilería los que fueron construidos utilizando como unidad de albañilería al ladrillo tipo Blocker II del grupo ladrillera Martorell de Tacna. Con los resultados de los ensayos y la recopilación de otros ensayos realizados en investigaciones en la universidad, se determinó valores de Fuerza Cortante $\left(f^{\prime} m\right)$ y Resistencia al Corte $\left(v^{\prime} m\right)$. En base a todos estos estudios se elaboró una propuesta de fórmula de la Densidad Mínima de Muros, la cual servirá para calcular la longitud mínima de muros que se necesita en una edificación de albañilería construida con muros portantes de blocker II para poder resistir un sismo severo.

Palabras Claves: Albañilería; Blocker II; Densidad de muros.

\section{Abstract}

This project was carried out under the financing of the "VI Competition of financing for research projects Private University of Tacna (UPT)". In this project, tests have been carried out on the piles and the death of masonry, which were built using the masonry unit to block II of the Martorell brickyard. With the results of the essays and the compilation of other essays carried out by our university students, the values of $f^{\prime} m$ and $v^{\prime} m$ have been determined. The base of all the studies has been elaborated a proposal of formula of the minimum density of the walls, which allows us to calculate the minimum length of the walls that is needed in a masonry building built with load-bearing walls of the blocker II so that it can resist a severe earthquake.

Keywords: Masonry; Blocker II; Density of walls. 


\section{Introducción}

El Perú se ubica dentro del cinturón de fuego del Pacífico o anillo del fuego del Pacífico, y cuya característica es que se concentra las zonas de subducción más importantes en el mundo y que han dado lugar a diversos terremotos. Después de ocurrido el sismo del 23 de Junio del 2001, los especialistas recomendaron a la población tacneña hacer uso de unidades sólidas y de resistencia adecuada. Sin embargo, los pobladores de Tacna aún vienen utilizando unidades huecas en la construcción de los muros portantes de sus viviendas como es el caso del Blocker II. Al respecto San Bartolomé (2005) indica que en la construcción de muros portantes confinados se debe emplear ladrillos que clasifiquen como unidades sólidas y que cumplan las especificaciones señaladas en la NTE E.070 ( 2006) de Albañilería. En muros portantes no debemos utilizar unidades huecas, ya que estas se trituran frágilmente después de que se generan las grietas diagonales en los muros. Por otro lado, en la tabla 2 de la NTE E.070 especifica que la unidad hueca no puede ser utilizada en construcción de muros portantes de edificaciones ubicadas en zonas altamente sísmicas como es el caso de la ciudad de Tacna. La norma citada indica la fórmula para determinar la densidad mínima de muros portantes en cada dirección de análisis, pero esta solo se aplica para unidades sólidas y de resistencia adecuada.

Para el caso del Blocker II, es necesario determinar las propiedades mecánicas y evaluar si se puede emplear esta fórmula, ya que su uso no está reglamentado en la NTE E-070, considerando que esta normatividad ha sido elaborada sólo para unidades macizas como es el caso del ladrillo Hércules I, no obstante más del 60 \% de las recientes construcciones en la ciudad de Tacna, son hechas haciendo uso del Blocker II.

\section{Objetivos}

El objetivo de este trabajo fue proponer una fórmula para determinar la densidad de muros construidos con el Blocker II, de tal manera que sea usado en la determinación de la vulnerabilidad sísmica y mitigación de daños de las viviendas informales de nuestra ciudad.

\section{Metodología}

\subsection{Descripción del Blocker II}

El Blocker II, es una unidad de albañilería, que se produce y fabrica en la Ladrillera Martorell ubicada en la ciudad de Tacna. Según las especificaciones técnicas, tiene las siguientes medidas: largo = $29 \mathrm{~cm}$, ancho $=12 \mathrm{~cm}$ y alto $=17 \mathrm{~cm}$, según la figura 1. 


\section{Figura 1}

Vista de la unidad Blocker II.

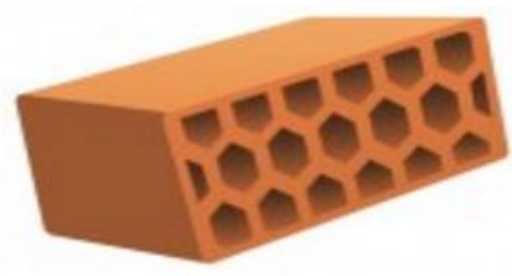

Nota: Medias: $12 \times 17 \times 29$. Uso para cercos perimétricos recintos amurallados y muros livianos. Rendimiento $17 \mathrm{unid} / \mathrm{m}^{2}$. Fuente: Ladrillera Martorell.

\subsection{Determinación de la Densidad de Muros según la NTE E.070}

En el ítem 19.2.b de la Norma Técnica de edificaciones (NTE) E.070 se especifica:

"La densidad mínima de muros portantes a reforzar en cada dirección del edificio se obtendrá mediante la siguiente expresión":

$$
\frac{\sum(\text { L.t })}{\mathrm{Ap}}=\frac{\text { Z.U.S.N }}{56}
$$

Dónde: Z, U y S corresponden a los factores de zona sísmica, Importancia y de suelo, respectivamente, especificados en la NTE E.030 Diseño Sismo resistente.

$\mathrm{N}=$ Número de pisos del edificio;

$\mathrm{L}=$ Longitud total del muro (incluyendo columnas, sí existiesen); $\mathrm{y}$,

$\mathrm{t}=$ Espesor efectivo del muro

De no cumplirse la expresión anterior podrá cambiarse el espesor de algunos de los muros, o agregarse placas de concreto armado, en cuyo caso, para hacer uso de la fórmula, deberá amplificarse el espesor real de la placa por la relación Ec / Em, donde Ec y Em son los módulos de elasticidad del concreto y de la albañilería, respectivamente. Asimismo, en comentarios a la Norma técnica de Edificaciones NTE E.070, el autor Ángel san Bartolomé menciona lo siguiente:

“En la fórmula (1) intervienen solo los muros reforzados con longitudes mayores que 1,2 m.

La fórmula (1) proviene de igualar la fuerza cortante actuante en la base del edificio ( $\mathrm{V}$, según la Norma E.030), a la resistencia al corte proporcionada por los muros orientados en la dirección en análisis $\left(\Sigma\left(v^{*} L^{*} t\right)\right)$. Para esto se supuso: un peso promedio de la planta típica (de área Ap) igual a 800 $\mathrm{kg} / \mathrm{m} 2$, una resistencia a fuerza cortante promedio $\mathrm{v}=3,7 \mathrm{~kg} / \mathrm{cm}^{2}\left(37000 \mathrm{~kg} / \mathrm{m}^{2}\right)$ en la albañilería; además, se admitió que este tipo de edificios (rígidos) cae en la zona plana del espectro sísmico, donde $C=2,5$, y que el factor de reducción de las fuerzas sísmica $(R)$ era igual a 3 , según se indica en la Norma E.030 para sismos severos que actúan en edificios de albañilería reforzada, con lo cual: 
Cortante actuante en la base (Norma E.030) $=\mathrm{V}$

$$
\mathrm{V}=\frac{\mathrm{Z} * \mathrm{U} * \mathrm{~S} * \mathrm{C} * \mathrm{P}}{\mathrm{R}}
$$

Y la resistencia al corte promedio (en rotura) es:

$$
\sum(\mathrm{v} * \mathrm{~L} * \mathrm{t})
$$

Igualando las ecuaciones (3) y (2) se obtiene:

$$
\begin{gathered}
37000 \mathrm{Kg} / \mathrm{m} 2 \sum \mathrm{L} * \mathrm{t}=\frac{\mathrm{Z} * \mathrm{U} * \mathrm{~S} * 2.5 *\left(800 \frac{\mathrm{Kg}}{\mathrm{m} 2} * \mathrm{Ap} * \mathrm{~N}\right)}{3} \\
111000 \sum(\mathrm{L} * \mathrm{t})=\mathrm{Z} * \mathrm{U} * \mathrm{~S} * 2000 * \mathrm{Ap} * \mathrm{~N} \\
\sum(\mathrm{L} * \mathrm{t})=\frac{\mathrm{Z} * \mathrm{U} * \mathrm{~S} * \mathrm{Ap} * \mathrm{~N}}{55.5} \\
\text { O } \quad \frac{\sum(\text { L.t })}{\mathrm{Ap}}=\frac{\text { Z.U.S.N }}{56}
\end{gathered}
$$

Cabe remarcar que una vez cumplida la fórmula (1), el resto de muros puede ser portante de carga vertical y carecer de confinamientos o de relleno total (muro armado parcialmente relleno), puesto que ante los terremotos los desplazamientos laterales de los muros no reforzados se encontrarán controlados por los muros portantes de carga sísmica (confinados o armados con relleno total), a través del diafragma rígido.

Por otro lado, en el ítem 8.5.4 de la NTE E.070 (Verificación de la resistencia al corte del edificio), se especifica: Con el objeto de proporcionar una adecuada resistencia y rigidez al edificio, en cada entrepiso i y en cada dirección principal del edificio, se deberá cumplir que la resistencia al corte sea mayor que la fuerza cortante producida por el sismo severo, es decir que:

$$
\Sigma \mathrm{V}_{\mathrm{mi}} \geq \mathrm{V}_{\mathrm{Ei}}
$$

La sumatoria de resistencias al corte $\left(\Sigma \mathrm{V}_{\mathrm{mi}}\right)$ incluirá sólo el aporte de los muros reforzados (confinados o armados) y el aporte de los muros de concreto armado, sin considerar en este caso la contribución del refuerzo horizontal.

El valor de $V_{\mathrm{Ei}}$ corresponde a la fuerza cortante actuante en el entrepiso i del edificio, producida por el "sismo severo". 


\section{Resultados}

En toda edificación de albañilería, una de las premisas importantes para su análisis está centrada en la densidad de muros que debe tener en ambas direcciones, es decir, que se analiza la cantidad de muros confinados existentes resistentes a sismo y se verifica si es que estos son capaces de resistir la fuerza cortante que se produce durante un evento sísmico.

En tal sentido, para proponer una nueva fórmula es necesario conocer las propiedades mecánicas de la unidad de albañilería en estudio. Es por ello que se realizaron ensayos en pilas y muretes efectuados en el laboratorio de estructuras del Centro Peruano Japonés de Investigaciones Sísmicas y Mitigación de Desastres (CISMID), de la Universidad Nacional de Ingeniería (UNI); y junto a la recopilación de datos de ensayos realizados en la Pontificia Universidad Católica del Perú se pudo determinar una resistencia al corte promedio, la cual fue utilizada para la determinación de la densidad de muros construidos con Blocker II.

\subsection{Ensayos en Pilas}

Con la finalidad de conocer las características del material frente a cargas de compresión axial y determinar su carga máxima y esfuerzo máximo se ensayaron 5 especímenes bajo carga incremental monotonía. Los especímenes denominados "pilas", son prismas de albañilería que se asientan uno sobre otro, teniendo como mínimo 3 unidades y como máximo 5 unidades.

Para la aplicación de la carga se utilizó una máquina de compresión SHIMATZU de una capacidad de 300 tf y la respuesta del espécimen se registró con un equipo de adquisición de datos, tal como se observa en la figura 2.

Se encontró que la resistencia a la compresión axial máxima promedio es de 48,37 kgf/cm2 y la desviación estándar es de 2,92 kgf/cm2, con lo cual se puede sugerir un valor de resistencia a la compresión f'm de 45,45 Kg/cm2.

\subsection{Ensayos en Muretes}

Este ensayo fue desarrollado para medir de una manera más precisa la tensión diagonal de la albañilería. El espécimen se carga en compresión a lo largo de una de sus diagonales provocando la falla por tensión diagonal en la dirección de aplicación de la carga, según se observa en la figura 3.

Se determinó que la resistencia al corte máximo promedio de $5,02 \mathrm{Kg} / \mathrm{cm}^{2}$ y la desviación estándar es de $0,83 \mathrm{Kg} / \mathrm{cm} 2$, por lo cual se puede sugerir una resistencia al cortante característico de $4,192 \mathrm{Kg} / \mathrm{cm}^{2}$. Asimismo, se obtuvo un valor promedio de módulo de cortante de $10565 \mathrm{Kg} / \mathrm{cm}^{2}$. 
Figura 2

Vista de los prismas a ser ensayado

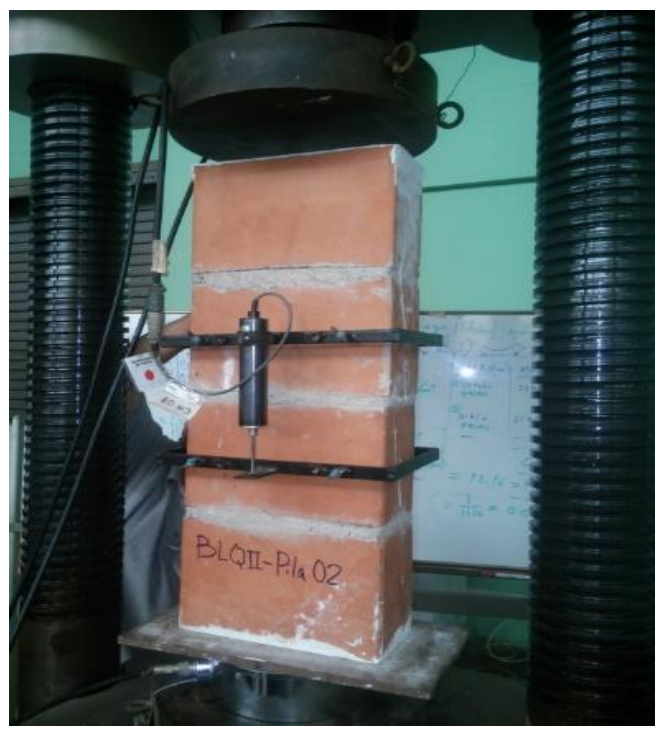

Nota. Ensayo realizado con máquina de compresión SHIMATZU de una capacidad de $300 \mathrm{tf}$.

Figura 3

Falla por tracción diagonal en las juntas y en los bloques

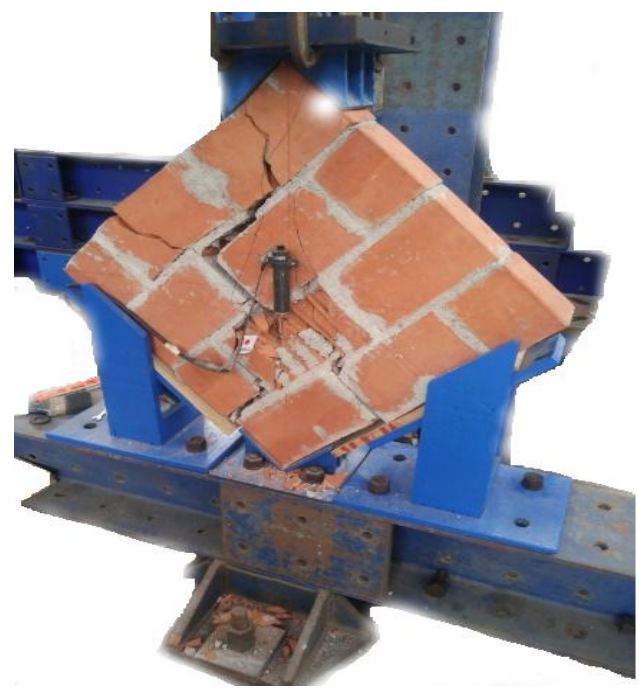

Nota. Imagen adaptada. 


\subsection{Resumen comparativo con otros trabajos de investigación y la NTE E 0.70}

A continuación, en la tabla 1 se muestra un resumen de los resultados obtenidos en los laboratorios del Centro Peruano Japonés de Investigaciones Sísmicas y Mitigación de Desastres (CISMID)-UNI y Pontificia Universidad Católica del Perú (PUCP). Se observa que existe variación en los resultados de un laboratorio con respecto a otro. Sin embargo, para ser más conservadores en nuestro análisis se tomará como resultado el promedio menos la desviación estándar.

De los resultados entre ambos laboratorios se puede observar que la resistencia a la compresión axial máxima promedio es de $64,035 \mathrm{Kg} / \mathrm{cm}^{2}$ y la desviación estándar es de $14,50 \mathrm{Kgf} / \mathrm{cm}^{2}$, con lo cual se puede sugerir un valor de resistencia a la compresión $f^{\prime} m$ de $49,53 \mathrm{Kg} / \mathrm{cm}^{2}$.

Asimismo, se determinó que la resistencia al corte máximo promedio de $4,10 \mathrm{Kg} / \mathrm{cm} 2$ y la desviación estándar es de $1,27 \mathrm{Kg} / \mathrm{cm}^{2}$, por lo cual se puede sugerir una resistencia al cortante característico de $2,834 \mathrm{Kg} / \mathrm{cm}^{2}$.

\begin{tabular}{|c|c|c|c|}
\hline Laboratorio & Identificación & $\begin{array}{c}v^{\prime} \mathrm{m} \\
\left(\mathrm{Kg} / \mathrm{cm}^{2}\right)\end{array}$ & $\begin{array}{c}\mathrm{G} \\
\left(\mathrm{Kg} / \mathrm{cm}^{2}\right)\end{array}$ \\
\hline \multirow{5}{*}{$\begin{array}{c}\text { CISMID UNI , (UPT, } \\
2016)\end{array}$} & BLQII-M01 & 4,36 & 10000 \\
\hline & BLQII-M02 & 5,64 & 13333 \\
\hline & BLQII-M03 & 5,78 & 10437 \\
\hline & BLQII-M04 & 5,39 & 10464 \\
\hline & BLQII-M05 & 3,92 & 8589 \\
\hline \multirow{4}{*}{$\begin{array}{c}\text { PUCP } \\
\text { (Aliaga y Vicente, } \\
\text { 2008) }\end{array}$} & M: 1 & 3,2 & 7956,94 \\
\hline & M: 2 & 3,28 & 10599,73 \\
\hline & M: 3 & 3,06 & 7449,3 \\
\hline & $M: 4$ & 2,28 & 8505,01 \\
\hline Promedio & & 4,101 & 9703,776 \\
\hline D.E & & 1,266 & 1802,469 \\
\hline Corregido & & 2,834 & 7901,306 \\
\hline
\end{tabular}

Nota. $\mathrm{v}^{\prime} \mathrm{m}$ resistencia al corte, en $\mathrm{Kg} / \mathrm{cm}^{2}$. G módulo de corte, en $\mathrm{Kg} / \mathrm{cm}^{2}$

.D.E desviación estándar

\subsection{Propuesta de fórmula para la determinación de la densidad de muros construidos con Blocker II}

En concordancia con el ítem 26.4.a de la NTE E.070 en donde se indica: Con el objeto de proporcionar una adecuada resistencia y rigidez al edificio, en cada entrepiso "i" y en cada dirección principal del 
edificio, se deberá cumplir que la resistencia al corte sea mayor que la fuerza cortante producida por el sismo severo, es decir que:

$$
\Sigma \mathrm{V}_{\mathrm{mi}} \geq \mathrm{V}_{\mathrm{Ei}}
$$

Para el primer piso:

$\mathrm{V}_{\mathrm{E}}=$ Fuerza cortante en la base calculado del análisis estático.

$\mathrm{V}_{\mathrm{m}}=$ Resistencia al corte de los muros

Además, los valores de la Fuerza Cortante en la base, están definidos en la Norma Técnica de Edificaciones E.030 (Diseño sismoresistente, 2003 y 2006) de la siguiente manera:

$$
\mathrm{VE}=\mathrm{V}=\frac{\mathrm{ZUSCP}}{\mathrm{R}}
$$

Dónde:

$Z=$ Factor de zona

$\mathrm{U}=$ Factor de uso

$\mathrm{S}=$ Factor de suelo.

C= Factor de amplificación sísmica

$\mathrm{R}=$ Factor de reducción

$\mathrm{P}=$ Peso de la estructura

Por otro lado, en el ítem 8.5.3 de la NTE E.070, se especifica que "la resistencia al corte (Vm) de los muros de albañilería se calculará en cada entrepiso mediante:

$$
V_{m}=0.5 v^{\prime} m \cdot \alpha \cdot t \cdot L+0.23 P_{g}
$$

Dónde:

$\mathrm{v}^{\prime} \mathrm{m}=$ resistencia característica al corte de la albañilería.

$\mathrm{P}_{\mathrm{g}}=$ carga gravitacional de servicio, con sobrecarga reducida de la NTE E.030 Diseño Sismoresistente.

$\mathrm{t}=$ espesor efectivo del muro.

$\mathrm{L}=$ Longitud total del muro (incluyendo a las columnas en el caso de muros confinados).

$\alpha=$ factor de reducción de resistencia al corte por efectos de esbeltez, calculado como:

$$
(1 / / 3) \leq V e . L / M e \leq 1
$$

$\mathrm{Ve}=$ Fuerza cortante del muro obtenida del análisis elástico $\mathrm{R}=6$.

$\mathrm{Me}=$ Momento flector del muro obtenido del análisis elástico $\mathrm{R}=6$.

La condición más desfavorable para la ecuación [5] es que ambos miembros sean iguales, por lo tanto:

$$
\Sigma \mathrm{V}_{\mathrm{m}} \cong \mathrm{V}_{\mathrm{E}}
$$


Debido a la variabilidad de valores que se podría tener para la ecuación [7], se ha despreciado el valor $(0,23 \mathrm{P}$ g), y se ha planteado también un valor conservador para el factor de reducción por esbeltez $(\alpha=1)$. Esta condición se ha establecido conociendo que la resistencia admisible al corte es la mitad de la resistencia a la rotura, es decir Vm es 0,5 v'm.L.t

Luego, rescribiendo la ecuación (8), tenemos:

$$
\sum\left(0.5 v^{\prime} m * L * t\right) \cong \frac{Z * U * S * C * P}{R},
$$

Ordenando:

$$
0.5 \mathrm{v}^{\prime} \mathrm{m} \sum(\mathrm{L} * \mathrm{t}) \cong \frac{\mathrm{Z} * \mathrm{U} * \mathrm{~S} * \mathrm{C} * \mathrm{P}}{\mathrm{R}}
$$

De manera similar a la fórmula 19.2.b de la NTE E.070, se puede asumir:

Un peso promedio de la planta típica de $800 \mathrm{~kg} / \mathrm{m}^{2}$; además, se puede suponer que para edificios rígidos, $\mathrm{C}$ cae en la zona plana del espectro sísmico, donde $\mathrm{C}=2,5$, y el factor de reducción de las fuerzas sísmica (R) es igual a 3. Con lo cual:

$$
\frac{\sum \mathrm{L} * \mathrm{t}}{\mathrm{Ap}} \cong \frac{\mathrm{Z} * \mathrm{U} * \mathrm{~S} * \mathrm{~N}}{0.00075 \mathrm{v}^{\prime} \mathrm{m}} ; \text { en } \frac{\mathrm{Kg}}{\mathrm{m} 2}
$$

En la tabla 1 de nuestro trabajo, se obtuvo un v'm igual a $2,834 \mathrm{Kg} / \mathrm{cm}^{2}$ o $28340 \mathrm{~kg} / \mathrm{m}^{2}$, entonces reemplazando este valor en (10), se obtiene la siguiente fórmula:

$$
\frac{\sum(\text { L.t })}{\text { Ap }} \geq \frac{\text { Z.U.S.N }}{22}
$$

Dónde: Z, U y S corresponden a los factores de zona sísmica, Importancia y de suelo, respectivamente, especificados en la NTE E.030 Diseño Sismo resistente.

$\mathrm{N}=$ Número de pisos del edificio;

$\mathrm{L}=$ Longitud total del muro (incluyendo columnas, sí existiesen); $\mathrm{y}$, $\mathrm{t}=$ Espesor efectivo del muro.

\section{Discusión}

Se presenta una propuesta de fórmula para la determinación de la densidad de muros en edificaciones construidas con blocker II (de la ladrillera Martorell), ya que a pesar que el fabricante indica que su uso es para cercos perimétricos, recintos amurallados y muros livianos, en la actualidad viene siendo utilizado en la construcción de muros portantes de las viviendas informales de la ciudad de Tacna. 
Comparando ambas fórmulas, el dividendo varia de 56 para unidades sólidas a 22 para el caso de la unidad hueca blocker II.

Si hacemos un comparativo en una edificación de 2 pisos, Con un área techada en planta de $88,00 \mathrm{~m}^{2}$, de configuración estructural regular en planta y elevación, y destinada al uso de vivienda unifamiliar, ubicada en el Distrito de Ciudad Nueva, Provincia y Departamento de Tacna, entonces se tiene:

- La longitud mínima necesaria según la NTE E.070 es: 13 metros lineales

- La longitud mínima necesaria según la propuesta de fórmula es: 33 metros lineales, es decir 275 $\%$ más que lo que se requiere en la NTE E.070.

En el año 2001, muchas de las construcciones presentaron fallas de corte, a pesar de ser construcciones de un sólo piso, esto se debe básicamente a la calidad del material empleado y a su baja resistencia. Mediante la aplicación de esta propuesta de fórmula, se puede identificar rápidamente la vulnerabilidad de la construcción ante un evento sísmico de gran magnitud. Sin embargo es conveniente realizar estudios experimentales en muros a escala real, así como propuestas de reforzamiento de las viviendas informales construidas con blocker II.

El Blocker II es una unidad hueca con un 53,93 \% de área hueca y según la tabla 2 de la Norma técnica de edificaciones E.070 (Albañilería, Limitaciones en el uso de la unidad de albañilería para fines estructurales), la unidad hueca no se puede utilizar en construcción de muros portantes para edificios ubicados en la zona sísmica 3, como es el caso de Tacna.

Se muestra un resumen de los resultados obtenidos en los laboratorios del CISMID-UNI y PUCP. De los resultados entre ambos laboratorios se determinó que la resistencia al corte máximo promedio de $4,10 \mathrm{Kg} / \mathrm{cm}^{2}$ y la desviación estándar es de $1,27 \mathrm{Kg} / \mathrm{cm}^{2}$, por lo que se puede sugerir una resistencia al cortante característico de $2,834 \mathrm{Kg} / \mathrm{cm} 2$ o $0,28 \mathrm{Mpa}$.

\section{Conclusión}

Para determinar la densidad mínima de muros construidos con Blocker II, y con techo rígido, se propone la siguiente expresión:

$$
\frac{\sum(\text { L.t })}{\mathrm{Ap}} \geq \frac{\text { Z.U.S.N }}{22}
$$

Dónde: Z, U y S corresponden a los factores de zona sísmica, Importancia y de suelo, respectivamente, especificados en la NTE E.030 Diseño Sismo resistente. N es el número de pisos del edificio; L es la longitud total del muro (incluyendo columnas, sí existiesen); $y, t$ es el espesor efectivo del muro 


\section{Referencias Bibliográficas}

Aliaga Rodriguez, I. J., \& Brañez Gonzales, V. M. (2009). Estudio comparativo del módulo de elasticidad en las unidades típicas de arcilla aplicado en el análisis sísmico de una vivienda unifamiliar del distrito de Ciudad Nueva del Departamento de Tacna, Tesis para optar el título profesional de Ingeniero Civil. Tacna: Universidad Privada de Tacna.

San Bartolomé, A., Qiunn, D. \& Silva, W. (2011). Diseño y construcción de estructuras sismoresistentes de albañilería. Fondo editorial PUCP. Lima.

Norma técnica de Edificaciones E.070, NTE E.070 (2006). Recuperado de http://blog.pucp.edu.pe/blog/wp-content/uploads/sites/82/2008/01/Norma-E-070-MV-2006.pdf Ladrillos Maxx. Recuperado de http://www.ladrillosmaxx.com/new/

Ángel San Bartolomé. (2005). Comentarios a la Norma Técnica de Edicación E . 070 Albañilería Informe Final. 147. http://blog.pucp.edu.pe/blog/albanileria/

Peruana, N. T. (2006). Norma técnica e.070 albañilería 2006 1. Ntp, 1-58.

\section{Agradecimientos}

A la Universidad privada de Tacna, por haber financiado el presente proyecto mediante el "VI Concurso de financiamiento para proyectos de Investigación Universidad Privada de Tacna (UPT)" y la Unidad de Investigación de la Facultad de Ingeniería de la misma universidad.

Al Mag. José Acero e Ingenieros; Daniella Aste, Claudia Ayca y Jhonatan Chávez, por su colaboración en la realización de los ensayos en la ciudad de Lima. 